\title{
Adenoid bacterial colonization in a paediatric population
}

\author{
João Subtil ${ }^{1} \cdot J_{0}$ ão Carlos Rodrigues ${ }^{2} \cdot$ Lúcia Reis $^{2} \cdot$ Luís Freitas $^{1} \cdot$ Joana Filipe $^{1}$.

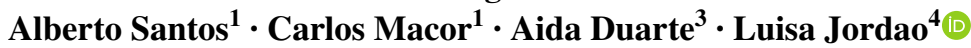

Received: 4 April 2016/ Accepted: 2 February 2017

(C) Springer-Verlag Berlin Heidelberg 2017

\begin{abstract}
Adenoids play a key role in both respiratory and ear infection in children. It has also been shown that adenoidectomy improves these symptoms in this population. The main goal of the present study was to evaluate adenoid bacterial colonization and document a possible relation with infectious respiratory disease. A prospective observational study was designed to evaluate the proposed hypothesis in a paediatric population submitted to adenoidectomy by either infectious or non-infectious indications and compare these two cohorts. A total of 62 patients with ages ranging from 1 to 12 years old were enrolled in the study. Adenoid surface, adenoid core and middle meatus microbiota were compared. A close association between adenoid colonization and nasal infection was found, supporting that adenoids may function as bacterial reservoir for upper airway infection. The obtained results also contribute to explain the success of adenoidectomy in patients with infectious indications.
\end{abstract}

Luisa Jordao

maria.jordao@insa.min-saude.pt

1 Serviço de otorrinolaringologia (ORL), Hospital de Beatriz Ângelo (HBA), Avenida Carlos Teixeira 3, 2674-514 Loures, Portugal

2 Departamento de Doenças Infeciosas (DDI), Instituto Nacional de Saúde Dr. Ricardo Jorge (INSA), Avenida Padre Cruz, 1649-016 Lisbon, Portugal

3 Departamento de Microbiologia e Imunologia, Faculdade de Farmácia da Universidade de Lisboa, Av Prof Gama Pinto, 1649-003 Lisbon, Portugal

4 Departamento de Saúde Ambiental, Unidade de Investigação e Desenvolvimento, INSA, Avenida Padre Cruz, 1649-016 Lisbon, Portugal
Keywords Adenoidectomy $\cdot$ Nasal and adenoidal microbiota $\cdot$ Upper airways infection $\cdot$ Children

\section{Introduction}

The adenoid tonsil plays a key role in respiratory disease in children, be it infectious or non-infectious [1]. In children, adenoid hypertrophy is associated with acute rhinosinusitis in $32.9 \%$ of the cases. On the other hand, there is a high coexistence of adenoid infection and nasal sinusitis especially in children with ages between 2- and 5-year-olds [1]. Also it has been shown that adenoidectomy improves symptoms of chronic sinusitis in children [1]. A potential explanation for these findings is the presence of biofilms on adenoid surface [2]. There is growing evidence that adenoid core bacteria share identity with the biofilm forming bacteria on the adenoid surface and in the middle meati, and may, therefore, be responsible for respiratory disease [3-5]. Knowing this, the present study aims to contribute to the body of knowledge, comparing bacterial population in both adenoid core and surface, and in the middle meati of children having adenoid surgery. Furthermore, the evaluation of a possible relationship between adenoid colonization and infectious respiratory disease, comparing two populations either with infectious and non-infectious surgical indication is also a goal of the study.

\section{Materials and methods}

\section{Study design and ethics}

A prospective observational study was designed to evaluate the importance of nasopharyngeal and adenoid 
colonization on ear and upper respiratory infections in a paediatric population of a tertiary Hospital in Greater Lisbon, Hospital de Beatriz Angelo (HBA).

The study was approved by HBA Medical Ethics Board in accordance with the World Medical Association Declaration of Helsinki. An informed consent was obtained from the children tutors to allow the collection of 3 different samples (nasal swab, superficial adenoid swab and adenoid core biopsy) for microbiota study. Data related to clinical and children status were also collected.

Opportunity sampling was used for a 1 year period, enrolling 62 children undergoing adenoid surgery, divided in two cohorts: children with an infectious diagnosis like recurrent acute upper airway infection (otitis media, adenoiditis, tonsillitis and sinusitis), chronic rhinosinusitis, adenoiditis or chronic purulent otitis media (Group 1); and children with non-infectious diagnosis (obstructive sleep apnoea, chronic otitis media with effusion--Group 2).

Nasal swabs were collected during surgery from the middle meati, while protecting from the nasal fossae mucosa using a sterile nasal speculum. Adenoid surface swab was collected after retracting the palatal velum providing frank and unobstructed access to the adenoid surface. Adenoid core sample was collected through the same path, after the first curettage provided access to the deeper adenoid layers, taking special care not to collect surface fimbriae on the raw surface.

\section{Bacterial isolation and identification}

The nasal and adenoidal swabs were inoculated into brain heart infusion (BHI) and the adenoidal biopsies into phosphate buffer saline (PBS) and processed within the next $8 \mathrm{~h}$. The swabs and biopsies were homogenised and plated on Columbia agar with 5\% sheep blood (COS) for isolation of non-fastidious bacteria; Chocolate PolyViteX agar (PVX) for isolation of fastidious bacteria; Chocolate PolyViteX VCAT3 agar (VCA3) for isolation of N. meningitidis; Mac Conkey agar (MC) for isolation of gram-negative bacilli; Columbia CNA agar with 5\% sheep blood (CNA) for isolation of staphylococci and streptococci and Schaedler agar with 5\% sheep blood (SCS) for isolation of anaerobic bacteria. The PVX and VCA3 were incubated at $37^{\circ} \mathrm{C}$ in a $5 \% \mathrm{CO}_{2}$ atmosphere; SCS was incubated at $37^{\circ} \mathrm{C}$ in an anaerobic atmosphere and all the others were incubated at $37^{\circ} \mathrm{C}$. In all the cases the cultures were incubated until $48 \mathrm{~h}$ being monitored after $18 \mathrm{~h}$. The bacteria were further cultivated to obtain pure cultures and were identified using VITEK2 (BioMérieux). All the culture media were purchased from BioMérieux.

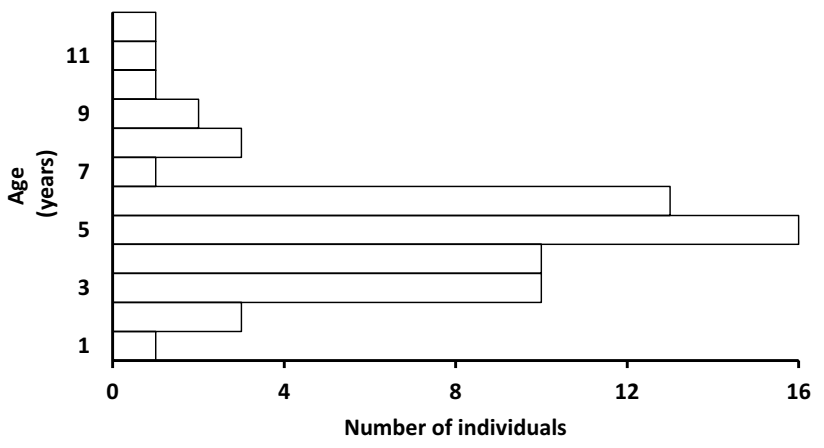

Fig. 1 Age distribution. The age distribution of the 62 individuals enrolled for the study is shown

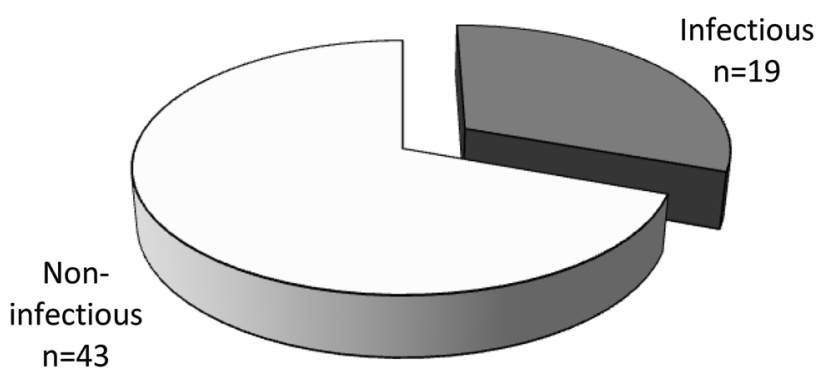

Fig. 2 Adenoidectomy indications. The population was divided in two cohorts according to the nature of the indication for adenoidectomy: a cohort of 19 infectious patients and another of 43 non-infectious

\section{Statistical analysis}

Unadjusted association between surgery indication and identity of the 4 major genera on nasal surface, adenoid surface and core microbiota were evaluated by Chi-square test. A $p$ value inferior to 0.05 was considered statistically significant.

\section{Results}

Population data: 62 patients were enrolled in the study ( 28 females and 34 males) with ages ranging between 1 and 12 years old (Fig. 1). The majority of the population (79.0\%) had between 3 and 6 years old. The mode corresponds to the age of 5 years old representing $25.8 \%$ of the individuals.

The population was divided in two cohorts according to surgery indication: Group $1(n=19)$ infectious indication and Group $2(n=43)$ non-infectious indication as shown in Fig. 2. In Group 1 the major surgery indications were recurrent acute upper airway infection (otitis media, sinusitis, adenoiditis, alone or associated with other diagnosis), chronic rhinosinusitis or chronic purulent otitis media; 
non-infectious diagnosis were obstructive sleep apnoea or chronic otitis media with effusion (Group 2). All the participants, with the exception of one, were not subjected to antibiotic therapy within the previous month to adenoidectomy.

A normal distribution was observed for the age of individuals enrolled for both groups with equal values of median, mode and average. Nevertheless, the age modes calculated were 6 and 5 years old for Group 1 and 2, respectively.

Microbiota: From 62 patients were taken 186 samples, including adenoid core, adenoid surface and nasal swab from each patient being identified 33 bacterial genera, including potential pathogenic bacteria such as Streptococcus pneumoniae, Haemophilus influenzae, beta-hemolytic streptococci, and Staphylococcus aureus. Commensal species, relatively stable flora members, enterococci, nonhemolytic streptococci, Neisseria spp., coagulase-negative staphylococci and Moraxella spp. were also found. On aerobic culture, the genera Haemophilus, Neisseria, Staphylococcus and Streptococcus were frequently isolated in both groups, and Group 2 population (non-infectious indications $n=43$ ) exhibits a higher bacterial diversity in all samples than Group 1 (infectious indication $n=19$ ). As shown in Table 1 Streptococcus spp. (40.35\%) and Staphylococcus spp. (19.38\%) were predominant for Group 1 and 2, respectively. Haemophilus genus was more frequent in Group 1 (19.3\%) than in Group $2(17.83 \%)$ being H. influenzae by far the most important species. $N$. meningitis was isolated only from one patient with obstructive sleep apnoea syndrome (Group 2). Commensal Neisseria species were found in $19.29 \%$ and $12.61 \%$ of samples from Group 1 and 2 , respectively.

Among 42 children (10 in Group 1 and 32 in Group 2 ), were simultaneously identified the same bacterial species on the adenoid core and at least one other sample (adenoid surface or nasal surface). The most representative species-H. influenzae, $S$. aureus, $S$. pneumoniae and S. pyogenes-were identified in 18 children simultaneously on the adenoid (core and surface) and on nasal samples; in contrast, from 14 children these species were isolated only on the adenoid core and surface. Group 2 exhibited a greater bacterial diversity in all samples than group 1 population (Fig. 3). From Group 1 nasal swabs were isolated 9 different bacterial species: S. aureus $(50 \%)$ and $H$. influenzae (30\%) the most frequent ones, followed by four species each with $10 \%$ (Fig. 3a). In Group 2, S. aureus (37.5\%) and H. influenzae (28.1\%) were also the most frequent, followed by Corynebacterium pseudodiphtheriticum, S. pneumoniae and other Streptococcus spp. each with $15.6 \%$ (Fig. 3b). In addition, seven other bacterial species were identified in lower percentages (Fig. 3b). The same trend of greater bacterial diversity in Group 2 than in Group 1 was observed
Table 1 Major microbiota genera

\begin{tabular}{lll}
\hline Bacteria & Group 1 (\%) & Group 2(\%) \\
\hline Haemophilus influenzae & 15.79 & 13.18 \\
Haemophilus parainfluenza & - & 2.33 \\
Haemophilus influenzaelparainfluenza & - & 0.78 \\
Haemophilus spp. & 3.51 & 1.55 \\
& 19.30 & 17.83 \\
Neisseria meningitidis & - & 0.78 \\
Neisseria cinerea & 1.75 & 3.10 \\
Neisseria lactamica & 1.75 & 0.78 \\
Neisseria sicca & 5.26 & 0.78 \\
Neisseria spp. & 10.53 & 7.75 \\
& 19.29 & 13.39 \\
Staphylococcus aureus & 15.79 & 14.73 \\
Staphylococcus cohnii & - & 0.78 \\
Staphylococcus epidermidis & 1.75 & 1.55 \\
Staphylococcus hominis & - & 2.33 \\
& 17.54 & 19.38 \\
Streptococcus alactolyticus & - & 0.29 \\
Streptococcus constellatus & - & 0.29 \\
Streptococcus cristatus/ sanguinis & 3.51 & - \\
Streptococcus pyogenes & 1.75 & 2.57 \\
Streptococcus Grupo C & 3.51 & 1.14 \\
Streptococcus hominis & - & 0.29 \\
Streptococcus intermedius & 1.75 & - \\
Streptococcus mitis/oralis & 8.77 & 3.15 \\
Streptococcus parasanguinis & - & 1.14 \\
Streptococcus plurianimalium & 5.26 & 0.29 \\
Streptococcus pneumoniae & 5.26 & 3.72 \\
Streptococcus pseudoporcinus & - & 0.86 \\
Streptococcus salivarius & 7.02 & 2.00 \\
Streptococcus sanguinius & 3.51 & 0.29 \\
& 40.35 & 16.01 \\
\hline & & \\
& & \\
& & \\
& &
\end{tabular}

both on adenoid surface (Fig. 3c, d) and core samples (Fig. 3e, f). A total of 8 and 14 different bacterial species were isolated from adenoid core and surface of Group1 and 2 , respectively. $H$. influenzae, S. aureus and other potential pathogens such as $S$. pneumoniae, S. pyogenes and N. meningitidis were identified in all the sub-sites on more than one sample.

A possible association between adenoid surface and nasal colonization and adenoid core invasion by bacteria was evaluated through chi-square test. In Group 1, a significant statistical associations was found for Haemophilus, Neisseria, Staphylococcus and Streptococcus genera on nasal and adenoid surface $(p<0.00001)$, on adenoid surface and core $(p<0.00001)$ and on the nasal surface and adenoid core $(p=0.000126)$. On the other hand, in Group 2 significant associations between nasal surface and both adenoid surface $(p<0.00001)$ and core $(p=0.000091)$ were 

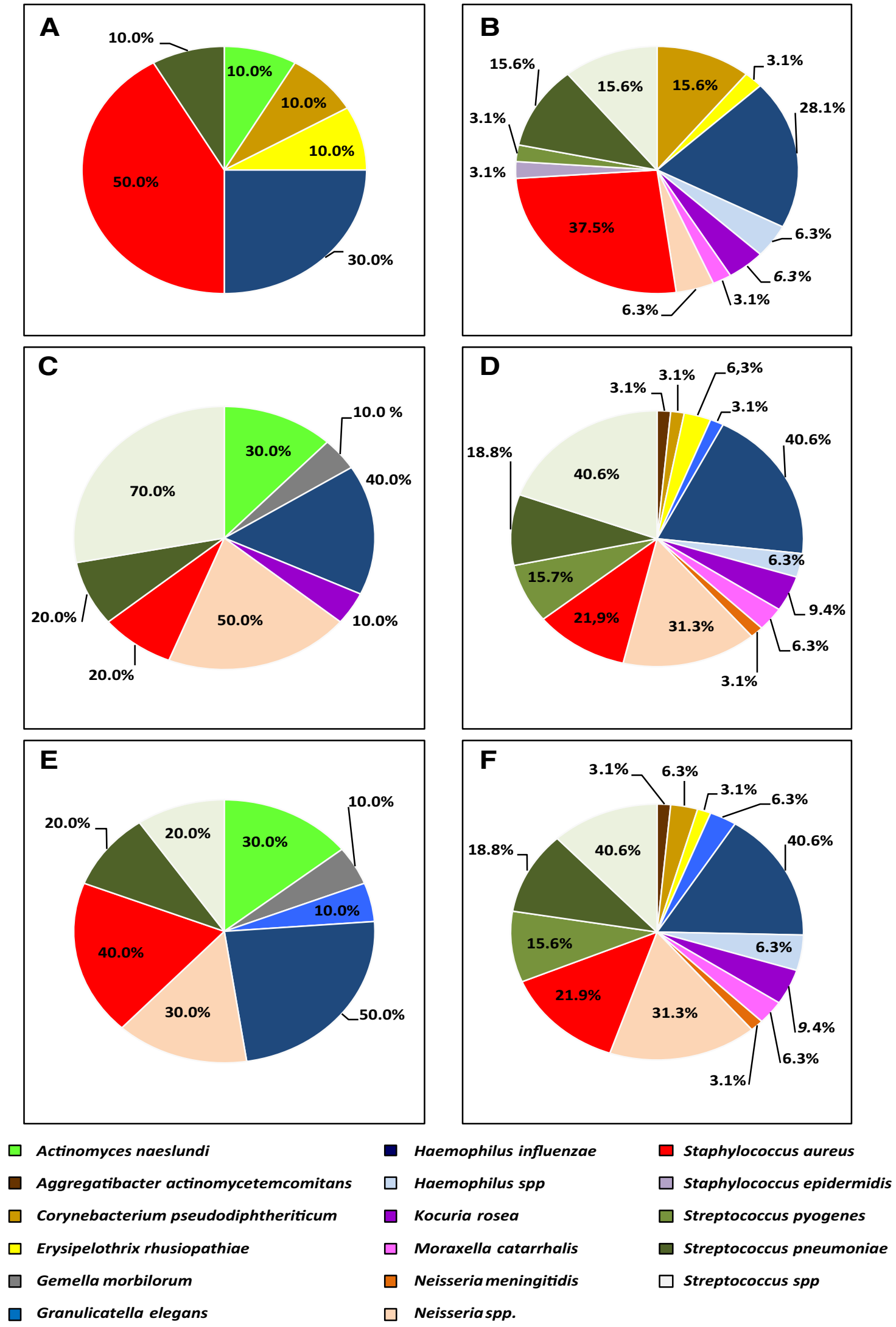

Fig. 3 Bacterial identity. The nasal, adenoid surface and core microbiota are shown in $\mathbf{a}, \mathbf{c}$ and $\mathbf{e}$ for Group 1 , respectively. In $\mathbf{b}, \mathbf{d}, \mathbf{f}$ is shown the microbiota of nasal, adenoid surface and core of Group 2 
found. No significant association was found between adenoid surface and core flora $(p=0.656)$, at least for the main genera of bacteria.

\section{Discussion}

The identity between adenoid tonsil and upper airway bacterial colonization is known for some time [1], mostly from indirect evidence or from bacterial studies after the adenoid tissue was removed from the patient [3-5]. In the present work this issue was overcame by collecting core samples directly in situ, after removing a fragment from the adenoid surface. Unlike the palatine tonsils, adenoid tonsils in most cases are not as thick and have an irregular surface, making it difficult to clearly separate core from surface sampling. For this reason all samples that were not thick enough to provide a safe distinction between the two sources were excluded. This procedure allowed avoiding bias in the results.

Surface colonization has recently gained importance after being described the possible involvement of surface biofilms on upper airway infections [2, 6-9]. Because of this, core colonization has lost relevance in the eyes of researchers, in contrast to what has been happening in palatine tonsils, where core bacteria are a major concern [10]. This knowledge is important, because when diagnosing tonsil disease, it is not possible to rely surely on surface swabs, and the same may apply to adenoid tonsil disease. Knowing that these interactions are of paramount importance in explaining the causality of adenoid and upper airway infection, it could be presumed that adenoids function as a reservoir for re-infection as previously postulated [3, 11]. The bacterial genera found in this study are similar to those previously described, namely Haemophilus, Staphylococcus and Streptococcus [3, 12]. Streptococcus was the most frequent bacteria found, therefore, associated with infectious diagnosis [1]. Finding Staphylococcus as the most frequent bacteria in non-infectious cases was also not a surprise, since it is ubiquitous and known for being a nasopharyngeal commensal [13].

What has emerged as unusual was the identification of $N$. meningitidis not reported as a relevant infectious agent neither in pharynx nor in nasal cavities, but is certainly related to other serious infections, namely meningitis [14]. For this reason, our finding deserves prompt attention. Although not usual, it has been previously reported [15] and is, in our mind, not an innocent bystander in these patients.

Other interesting result was finding no statistically significant association between adenoid surface and adenoid core flora, at least for the main genera of bacteria, and this pairs with the majority of previous works on the palatine tonsils [5].
These results, therefore, come in line with previous reports describing that surface colonization in the adenoid tonsils may play a role in upper airway infections. This has medical and surgical therapeutical implications, for it contributes to explain the beneficial role of nasal irrigation $[1,16]$ and of adenoidectomy in children with these chronic infections [1].

In this study, the severity of the colonization was not quantified, since none of the participants were acutely inflamed, and therefore, bacteria were considered colonists and not infectious. To rule out adenoid inflammation the previously established criterion of mucous observed over the adenoid mucosa was used [15]. As such, the colonization was not quantified, and therefore, the bacteria were considered commensal instead of potentially pathogenic.

The most important limitation of this study was the number of participants and the asymmetry of the groups, with infectious indications (Group 1) being less common. Nevertheless, given the relative scarcity of literature on the subject, and also the innovative sampling method for the adenoid core, it is an important contribution for the state of the art.

\section{Conclusion}

A close association between adenoid surface colonization and nasal infection was found supporting the concept of adenoid tonsil as a bacterial reservoir for upper airway infection. This also contributes to explain the success of adenoidectomy in cases with infectious indications. Other interesting result was finding no significant association between adenoid surface and core flora, at least for the main genera of bacteria.

Acknowledgements The authors thank to Nacional de Saúde Dr. Ricardo Jorge for financial support (Grant 2015DDI1143), to research nurses Sofia Simoes and Claudia Vieira from HBA for technical support during the sample collection stage of the study.

\section{Compliance with ethical standards}

Conflict of interest The authors declare no conflict of interest.

Funding Grant 2015DDI1143 attributed to Luisa Jordao and Joao Carlos Rodrigues by the Nacional de Saúde Dr. Ricardo Jorge was used to support the present work.

Ethical approval The study was approved by Hospital de Beatriz Ângelo Medical Ethics Board in accordance with the World Medical Association Declaration of Helsinki. 


\section{References}

1. Fokkens WJ, Lund VJ, Mullol J, Bachert C, Alobid I, Baroody F, Cohen N, Cervin A, Douglas R, Gevaert P, Georgalas C, Goossens H, Harvey R, Hellings P, Hopkins C, Jones N, Joos G, Kalogjera L, Kern B, Kowalski M, Price D, Riechelmann H, Schlosser R, Senior B, Thomas M, Toskala E, Voegels R, Wang DY, Wormald PJ (2012) European position paper on rhinosinusitis and nasal polyps. Rhinology Suppl. 23:1-299

2. Zuliani G, Carron M, Gurrola J, Coleman C, Haupert M, Berk R, Coticchia J (2006) Identification of adenoid biofilms in chronic rhinosinusitis. Int J Pediatr Otorhinolaryngol 70:1613-1617. doi:10.1016/j.ijporl.2006.05.002

3. Elwany S, El-Dine AN, El-Medany A, Omran A, Mandour Z, El-Salam AA (2011) Relationship between bacteriology of the adenoid core and middle meatus in children with sinusitis. J Laryngol Otol 125:279-281. doi:10.1017/S0022215110002586

4. Kosikowska U, Korona-Głowniak I, Niedzielski A, Malm A (2015) Nasopharyngeal and adenoid colonization by Haemophilus influenzae and Haemophilus parainfluenzae in children undergoing adenoidectomy and the ability of bacterial isolates to biofilm production. Medicine (Baltimore) 94:e799. doi:10.1097/ MD.0000000000000799

5. Taylan I, Ozcan I, Mumcuoğlu I, Baran I, Murat Özcan K, Akdoğan O, Selcuk A, Balaban N, Dere H (2011) Comparison of the surface and core bacteria in tonsillar and adenoid tissue with beta-lactamase production. Indian. J Otolaryngol Head Neck Surg 63:223-228. doi:10.1007/s12070-011-0265-Z

6. Blanchette KA, Orihuela CJ (2012) Future perspective on host-pathogen interactions during bacterial biofilm formation within nasopharynx. Future Microbiol 7:227-239. doi:10.2217/ fmb.11.160

7. Torretta S, Drago L, Marchisio P, Mattina R, Clemente IA, Pignataro L (2011) Diagnostic accuracy of nasopharyngeal swabs in detecting biofilm-producing bacteria in chronic adenoiditis: a preliminary study. Otolaryngol Head Neck Surg 144:784-788. doi:10.1177/0194599810394955
8. Drago L, De Vecchi E, Torretta S, Mattina R, Marchisio P, Pignataro L (2012) Biofilm formation by bacteria isolated from upper respiratory tract before and after adenotonsillectomy. APMIS 120:410-416. doi:10.1111/j.1600-0463.2011.02846

9. Nazzari E, Torretta S, Pignataro L, Marchisio P, Esposito S (2015) Role of biofilm in children with recurrent upper respiratory tract infections. Eur J Clin Microbiol Infect Dis 34:421-429. doi:10.1007/s10096-014-2261-1

10. Singh K, Nordström T, Mörgelin M, Brant M, Cardell LO, Riesbeck K (2014) Haemophilus influenzae resides in tonsils and uses immunoglobulin D binding as an evasion strategy. J Infect Dis 209:1418-1428. doi:10.1093/infdis/jit593

11. Niedzielski A, Korona-Glowniak I, Malm A (2013) High prevalence of Streptococcus pneumoniae in adenoids and nasopharynx in preschool children with recurrent upper respiratory tract infections in Poland-distribution of serotypes and drug resistance patterns. Med Sci Monit 19:54-60. doi:10.12659/MSM.883742

12. Swidsinski A, Göktas O, Bessler C, Loening-Baucke V, Hale LP, Andree H, Weizenegger M, Hölzl M, Scherer H, Lochs H (2007) Spatial organisation of microbiota in quiescent adenoiditis and tonsillitis. J Clin Pathol 60:253-260. doi:10.1136/ jcp.2006.037309

13. Berry S, Morton S, Atatoa Carr P, Marks E, Ritchie S, Upton A, Williamson D, Grant C (2015) Colonisation with Staphylococcus aureus and Streptococcus pyogenes in New Zealand preschool children. N Z Med J 128:60-67

14. Dwilow R, Fanella S (2015) Invasive meningococcal disease in the 21st century-an update for the clinician. Curr Neurol Neurosci Rep 15:2. doi:10.1007/s11910-015-0524-6

15. Marseglia GL, Pagella F, Klersy C, Barberi S, Licari A, Ciprandi G (2007) The 10-day mark is a good way to diagnose not only acute rhinosinusitis but also adenoiditis, as confirmed by endoscopy. Int J Pediatr Otorhinolaryngol 71:581-583. doi:10.1016/j. ijporl.2006.12.003

16. Harvey R, Hannan SA, Badia L, Scadding G (2007) Nasal saline irrigations for the symptoms of chronic rhinosinusitis. Cochrane Database Syst Rev 18:CD006394 